\title{
Computational Intelligence Approach for Process Parameter Settings Using Knowledge Representation
}

\author{
Henry C. W. Lau
}

\begin{abstract}
This study proposes a fuzzy approach which integrates fuzzy rule sets in a chromosome. To enhance the functionality and capability of the fuzzy set, Genetic Algorithms (GA) technique is incorporated to produce a better and improved fuzzy set which is able to generate the expected result. Past data were selected to create the chromosomes and form the primary population set. This approach capitalizes on the merits of both techniques and offsets the drawbacks of them which may undermine the performance. This research signifies the hybrid approach to identify the optimal criteria for process control in order to achieve the target of the whole operations with an innovative methodology that has not been covered adequately to-date. A case example has been conducted to validate the practicality of the approach and the outcome demonstrated that the proposed approach is able to achieve the results as expected.
\end{abstract}

Index Terms-Genetic algorithms, demand uncertainty, supply chain management, fuzzy logic model.

\section{INTRODUCTION}

Knowledge and innovation are very important for potential business process improvements in a supply chain network with the increasing trends of short product life cycle and mass customization [1]. In order to improve the business process, lots of scholars focus on the process capability studies recently. The product features are measured and analyzed to determine the process ability process in order to meet product specifications [2]. Providing products and service in time with good quality is vital for business success. But how can a company optimize business processes to provide better products and service? Many scholars put great efforts in exploring the artificial intelligence (AI) techniques, such as intelligent systems [3].

How to develop a next generation intelligent system emerges as a new research interest for AI research. For example, some scholars focus on the integration of Genetic Algorithms (GA) and Fuzzy Logic approach [4]. However, the application of this hybrid approach is limited. This study proposes a Fuzzy GA approach to integrate fuzzy rule sets and their associated membership function sets into GA approach. With this hybrid approach, both crisp and fuzzy data can be integrated into one chromosome which is impossible in traditional GA approach.

The hybrid fuzzy-GA approach proposed in this study is conducted as follows. First, process parameter is encoded as a fuzzy rule sets and the fuzzy membership function is

Manuscript received February 16, 2014; revised May 6, 2014.

Henry C. W. Lau is with School of Business, University of Western Sydney, Australia (e-mail: H.lau@uws.edu.au). created for the fuzzy rule sets. Past data are selected to create the chromosomes and form the primary population set. Second, GA technique is used to produce a better and improved fuzzy set which is able to generate the expected result.

This paper has 7 sections. In Section II, literature concerning the fuzzy logic approach, GA approach, and their related applications are presented. Section III is about the 5 parts of the chromosome and proposed approach. Section IV presents the processes of GA approach, including crossover, mutation process, and the fitness function. A case example has been conducted in Section V to validate the practicality of the approach and the outcome in Section VI demonstrated that the proposed approach is able to achieve the results as expected. Finally, there is a brief conclusion in Section VII.

\section{LITERATURE REVIEW}

GA is one of the most popular search algorithms used by scholars in areas such as industrial engineering to attain a more accurate and optimal optimization result [5], [6]. For example, GA approach has been used to optimize industrial processes, reduce production costs, minimize manufacturing times and increase flexibility of machine selection [7]. GA approach has become a genetic optimization technique with machine learning capability. However, the GA algorithm has shown its weakness such as the preset value of the parameters could lead to premature convergence.

Integrating GA approach with other approaches, such as the Fuzzy logic approach, has become an emerging and promising research direction [8]. The fuzzy expert systems method has the capability of capturing that tacit knowledge that sits within the field experts, those who have experience from similar situations. The process involves studying and understanding the situation in order to design the relevant appropriate fuzzy system identifying the appropriate expertise and input data types. The core fuzzy system consists of the three stages of fuzzification, fuzzy inference engine and defuzzification . Benefits of the application of fuzzy logic principles are mainly in dealing with the uncertainties and ambiguities in the decision-making process [9].

Reactive Ion Etching (RIE) is a combination of chemical and physical dry etching. AI techniques were adopted to monitor the etching operation as the RIE process is highly nonlinear and multivariable [10]. However, literature review has shown that even though some approaches were used to optimize the RIE system, more research is still needed to explore the parametric settings for the RIE processes. This study addressed this issue and adopted the fuzzy-GA approach to solve this problem. 


\section{Process PARAmeter SetTING Model}

The proposed hybrid fuzzy-GA approach for RIE process optimization encodes customer requirements, process parameters and defect rate which are in a fuzzy rule set into one chromosome of the GA approach. The chromosome consists of 5 parts:

1) Customer requirement $\left(r_{i u}\right)$;

2) Process parameter $\left(p_{i v}\right)$;

3) Defect rate $\left(d_{i x}\right)$;

4) Fuzzy membership function of process parameter $\left(k_{i y}\right)$;

5) Fuzzy membership function of defect rate $\left(q_{i z}\right)$.

GA technique is used to produce a better and improved fuzzy set which is able to generate the expected result. The fitness function of the chromosome is derived from the fuzzy rule sets and evaluated based on the accuracy with error rate and the performance trade-off.

\section{FuZZY KNOWLEDGE REPRESENTATION IN A GENETIC} ALGORITHM

\section{A. Notations and Definitions}

Definition 1: $C_{h}=\{1,2, \ldots, M\}$ is the index set of chromosomes. $M$ is the total number of chromosomes in the population.

Definition 2: $G_{m \times w}$ is a $m \times w$ gene matrix generated for the population.

$$
\begin{aligned}
& G_{m \times w}=\left[\begin{array}{cccc|cccc|cccc|cccc}
p_{11} & p_{12} & \ldots & p_{1 b} & d_{11} & d_{12} & \ldots & d_{1 s} & k_{11} & k_{12} & \ldots & k_{1 e} & q_{11} & q_{12} & \ldots & q_{1 n} \\
p_{21} & p_{22} & \ldots & p_{2 b} & d_{21} & d_{22} & \ldots & d_{2 s} & k_{21} & k_{22} & \ldots & k_{2 e} & q_{21} & q_{22} & \ldots & q_{2 n} \\
\vdots & \vdots & \ddots & \vdots & \vdots & \vdots & \ddots & \vdots & \vdots & \vdots & \ddots & \vdots & \vdots & \vdots & \ddots & \vdots \\
p_{m 1} & p_{m 2} & \ldots & p_{m b} & d_{m 1} & d_{m 2} & \ldots & d_{m s} & k_{m 1} & k_{m 2} & \ldots & k_{m e} & q_{m 1} & q_{m 2} & \ldots & q_{m n}
\end{array}\right] \\
& =\left(\begin{array}{llll}
\left(p_{i v}\right)_{m \times b} & \left(d_{i x}\right)_{m \times s} & \left(k_{i j}\right)_{m \times e} & \left(q_{i z}\right)_{m \times n}
\end{array}\right) \\
& w=b+s+e+n \\
& p_{i v}=\operatorname{random}\left[l_{P_{p}}, u_{P_{p}}\right] \text {, } \\
& d_{i x}=\operatorname{random}\left[l_{D_{r}}, u_{D_{r}}\right] \text {, } \\
& k_{i, \tau}=c_{p_{i v}}, k_{i, \lambda}=w_{p_{i v}} \text {, } \\
& q_{i, \tau}=c_{d i x}, q_{i, \lambda}=w_{d i x}
\end{aligned}
$$

$\forall i \in C_{h}, \forall v \in P, \forall x \in D, \forall y \in A, \forall z \in B, \tau=1,3,5, \ldots \ldots ; \lambda=2,4,6, \ldots \ldots ;$ $m=M, b=P_{p}, s=D_{r}, e=6 P_{p}, n=6 D_{r}$

Definition 3: $B_{m \times 1}$ is a random number matrix. It is generated for the selection and crossover process.

$$
\begin{gathered}
B_{m \times 1}=\left[\begin{array}{c}
b_{1} \\
b_{2} \\
\vdots \\
b_{m}
\end{array}\right]=\left(b_{i}\right)_{m \times 1} \\
b_{i}=\operatorname{random}[0,1], \forall i \in C_{h}, m=M .
\end{gathered}
$$

Definition 4: $C_{h_{-} c}=\{1,2, \ldots \ldots, S\}$ represents the index for chosen chromosomes in the crossover process. $S$ is the total number of chosen chromosomes.

Definition 5: $G_{m \times w}^{\prime}$ is the $m \times w$ gene matrix where the $Q$ chromosomes chosen in crossover process are stored.

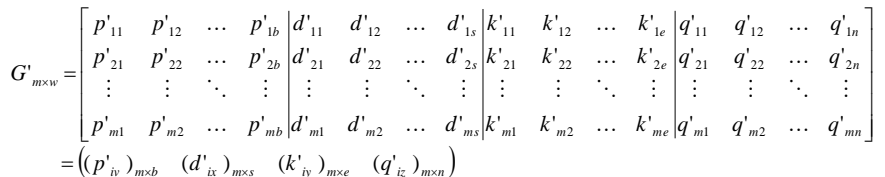

$$
\begin{aligned}
& w=b+s+e+n \\
& p_{i v}^{\prime}=\operatorname{random}\left\lfloor l_{P_{p}}, u_{P_{p}}\right\rfloor \\
& d_{i x}^{\prime}=\operatorname{random}\left\lfloor l_{D_{r}}, u_{D_{r}}\right] \\
& k_{i, \tau}^{\prime}=c_{p_{i v}}, k_{i, \lambda}^{\prime}=w_{p_{i v}}, q_{i, \tau}^{\prime}=c_{d i x}, q_{i, \lambda}^{\prime}=w_{d i x}, \\
& \forall i \in C_{h_{-} c}, \forall v \in P, \forall x \in D, \forall y \in A, \forall z \in B, \tau=1,3,5, \ldots \ldots ; \lambda=2,4,6, \ldots \ldots ; \\
& m=S, b=P_{p}, s=D_{r}, e=6 P_{p}, n=6 D_{r}
\end{aligned}
$$

Definition 6: mask $_{1 \times w}$ represents a mask matrix generated for crossover process.

$$
\begin{aligned}
& \operatorname{mask}_{1 \times w}=\left[m_{p_{1}} \ldots m_{p_{b}}\left|m_{d_{1}} \ldots m_{d_{s}}\right| m_{k_{1}} \ldots m_{k_{e}} \mid m_{q_{1}} \ldots m_{q_{n}}\right] \\
& =\left(\begin{array}{llll}
\left(m_{p_{v}}\right)_{1 \times b} & \left(m_{d_{x}}\right)_{1 \times s} & \left(m_{k_{y}}\right)_{1 \times e} & \left(m_{q_{z}}\right)_{1 \times n}
\end{array}\right) \\
& m_{p_{v}}=\operatorname{random}\{0,1\}, m_{d_{x}}=\operatorname{random}\{0,1\}, m_{k_{y}}=\operatorname{random}\{0,1\}, \\
& m_{q_{z}}=\operatorname{random}\{0,1\} \\
& \forall v \in P, \forall x \in D, \forall y \in A, \forall z \in B, b=P_{p}, s=D_{r}, e=6 P_{p}, n=6 D_{r}
\end{aligned}
$$

\section{B. Initial Population}

The proposed hybrid Fuzzy-GA approach is used to optimize the fuzzy rule set (i.e. If $p_{i v}$, then $d_{i x}$ ) where the associated fuzzy membership are $\tilde{F_{p_{i v}}}$ and $\tilde{F_{d_{i x}}}$. The initial population in the GA approach was generated through two approaches:

First, half of the total number of chromosomes was randomly generated by the computer;

Second, half of the total number of chromosomes was acquired from historical data (such as company's database, customer specifications, other similar companies, and data from associations) and experts' past experience.

\section{Uniform Crossover}

The GA approach is an iterative process. By using the crossover and mutation operators, new offspring are generated and appropriate replacements are conducted by the generated offspring solutions to create a new generation. For the crossover process, this study adopted the uniform crossover algorithm. The operation of uniform crossover 
process is shown in Fig. 1.

\begin{tabular}{|c|c|c|c|c|c|c|c|c|c|c|c|c|c|c|c|c|c|}
\hline & & & & & & & & & & & & & & & & & \\
\hline Mask & 1 & 0 & 1 & 0 & 0 & 0 & 1 & $\cdots$ & 0 & 1 & 1 & 0 & 0 & 1 & 0 & 1 & 0 \\
\hline Parent 1 & 3 & 4 & 2 & 3 & 0 & 0 & 1 & $\cdots$ & 2 & 5 & 2 & 3 & 5 & 1 & 6 & 1 & 7 \\
\hline Parent 2 & 2 & 1 & 1 & 3 & 1 & 0 & 2 & $\cdots$ & 2 & 8 & 3 & 7 & 3 & 2 & 6 & 2 & 3 \\
\hline rossove & & & & & & & & & & & & & & & & & \\
\hline Offspring 1 & 2 & 4 & 1 & 3 & 0 & 0 & 2 & $\cdots$ & 2 & 8 & 3 & 3 & 5 & 2 & 6 & 2 & 7 \\
\hline Offspring 2 & 3 & 1 & 2 & 3 & 1 & 0 & 1 & $\cdots$ & 2 & 5 & 2 & 7 & 3 & 1 & 6 & 1 & 3 \\
\hline
\end{tabular}

Fig. 1. Uniform crossover process.

The new offspring are then for the mutation process. The mutation process is shown in Fig. 2. The new offspring should be modified by exchanging $k_{1}$ and $k_{3}$ since

$$
\tilde{F_{p_{i v}}}=\left\{c_{p_{i v}}-w_{p_{i v}}, w_{p_{i v}}, c_{p_{i v}}, w_{p_{i v}}, c_{p_{i v}}+w_{p_{i v}}, w_{p_{i v}}\right\}(1)
$$

As shown in Fig. $2, k_{2}$ is modified by subtracting $k_{1}$ from $k_{3}$ and $k_{4}$ is modified by subtracting $k_{3}$ from $k_{5}$.

Offspring 1:

\begin{tabular}{llll|l|l|l|l|l|l|l|l|l|l|l|l|l|}
2 & 4 & 1 & 3 & 0 & 0 & 2 & $\cdots$ & 2 & 8 & 3 & 3 & 5 & 2 & 6 & 2 & 7 \\
Modification of offspring $1:$
\end{tabular}
\begin{tabular}{|lllllll|l|l|l|l|l|l|l|l|l|l|l|}
\hline 2 & 3 & 1 & 4 & 1 & 0 & 2 & $\cdots$ & 2 & 8 & 3 & 3 & 5 & 2 & 6 & 2 & 7 \\
\hline
\end{tabular}

Fig. 2. Modification after crossover.

\section{Fitness Function and Selection}

For each population of the GA process, the objective function values are evaluated to compute the level of fitness of each possible solution. Some individual solutions are randomly selected for reproduction where the probability of selection is proportional to their level of fitness. The fitness function in this study is to optimize the accuracy of the fuzzy rules and the performance trade-off function. The fitness function in formulation (2) is used to evaluate the derived fuzzy rule set. The objective of the fitness function is to maximize the accuracy and minimize the performance trade-off.

$$
\text { Fitness Funtion }=\frac{\text { Accurancy with error rate }}{\text { Performance tradeoff }}
$$

\section{CASE Examples of KnOWledge Assimilation}

A case example is conducted in the RIE process optimization in this section to validate the practicality of the hybrid fuzzy GA approach.

In the fuzzy inference engine, the fuzzy rules represented in the form of IF-THEN rules are developed based on the expert knowledge and historical data. This study forms the IF-THEN rules between defect rate and process parameter in RIE process, based on historical data and engineers' knowledge.

Table I is the customer requirements for the production of silicon chips. Based on the knowledge extracted from the company's historical database, a sample IF-THEN fuzzy rule is formulated.

If the pressure is high And RF power is medium And the usage of $\mathrm{CHF}_{3}$ is medium And the usage of $\mathrm{O}_{2}$ is high, THEN the vacuum defect E11 will be low and the vacuum defect E3 will be high.

The fuzzy rule set is then encoded into a chromosome. For each fuzzy rule set, the fitness value can be calculated using formulation (2), and the optimum fuzzy rule set can be found based on formulation (2).

\begin{tabular}{|c|c|c|}
\hline \multirow{2}{*}{$\begin{array}{l}\text { RIEC Customer } \\
\text { requirement }\end{array}$} & Etch Depth (D) & $12550 \mathrm{~nm}$ \\
\hline & $\begin{array}{l}\text { Shallow Specification } \\
\text { (W) }\end{array}$ & $1450 \mathrm{~nm}$ \\
\hline \multirow[t]{7}{*}{$\begin{array}{l}\text { RIE process } \\
\text { parameter }\end{array}$} & Pressure (PR) & $\begin{array}{l}\text { 5-15mTorr } \\
\text { Ignore (0) Low (1) } \\
\text { Medium (2) High (3) }\end{array}$ \\
\hline & RF Power (RFP) & $\begin{array}{l}\text { 50- } 150 \text { Watts } \\
\text { Ignore } \quad(0) \quad \text { Low (1) } \\
\text { Medium (2) High (3) }\end{array}$ \\
\hline & $\mathrm{CHF}_{3}$ & $\begin{array}{l}\text { 1- } 60 \mathrm{sccm} \\
\text { Ignore } \quad(0) \quad \text { Low (1) } \\
\text { Medium (2) High (3) }\end{array}$ \\
\hline & $\overline{\mathrm{O}_{2}}$ & $\begin{array}{l}\text { 1-60 sccm } \\
\text { Ignore } \quad(0) \quad \text { Low(1) } \\
\text { Medium (2) High (3) }\end{array}$ \\
\hline & Wall Angle(WA) & $\begin{array}{l}19-26 \\
\text { Ignore } \quad(0) \quad \text { Low }(1) \\
\text { Medium (2) High (3) }\end{array}$ \\
\hline & Cycle Time(CT) & $\begin{array}{l}\text { 1 - } 1.5 \text { hour } \\
\text { Ignore } \quad(0) \quad \text { Low }(1) \\
\text { Medium (2) High (3) }\end{array}$ \\
\hline & $\begin{array}{l}\text { Airgroove Roughness } \\
\text { (AR) }\end{array}$ & $\begin{array}{l}35-\quad 60 \mathrm{~nm} \\
\text { Ignore } \quad(0) \quad \text { Low }(1) \\
\text { Medium (2) High (3) }\end{array}$ \\
\hline \multirow[t]{2}{*}{ Defect } & vacuum defect E11 & $\begin{array}{l}5 \%-10 \% \\
(0) \text { Low }(1) \quad \text { Medium } \\
\text { High }\end{array}$ \\
\hline & vacuum defect E3 & $\begin{array}{l}5 \%-10 \% \\
(0) \operatorname{Low}(1) \quad \text { Medium } \\
\text { High }\end{array}$ \\
\hline
\end{tabular}

TABLE I: FUZZY TERM OF RIE PROCESS PARAMETER

\section{Testing Instance Based on Accuracy}

We assume that there is only one fuzzy set rule in the chromosome $_{1}$ and the output of E11 and E3 are both 7.5 based on expertise advice.

The ideal case (\#) for E11 ( $\left.y^{\prime}\right)=7.5$ and E3 is 7.5, $n=4$, weight of $\mathrm{E} 11=0.6$ and $\mathrm{E} 13=0.4$

Error rate is calculated based on the data of Table II.

Error rate $(\varepsilon)=$

$\sum_{j=1}^{m} w_{j} \frac{\left(y_{j}-y_{j}{ }^{\prime}\right)^{2}}{2 n}=\frac{0.6\left(0^{2}+0^{2}+1.28^{2}+1.32^{2}\right)+0.4\left(0^{2}+0^{2}+1.28^{2}+1.32^{2}\right)}{2 \times 4}$ $=0.4226$

TABLE II: EXTRACTED PAST RECORD TO FIND OUT THE ERROR RANGE

\begin{tabular}{|l|l|l|l|l|l|l|l|l|l|}
\hline \multicolumn{9}{|c|}{ Input } \\
\hline \begin{tabular}{l}
\hline Pressure \\
(PR)
\end{tabular} & $\begin{array}{l}\text { RF } \\
\text { Power(RFP) }\end{array}$ & $\mathrm{CHF}_{3}$ & $\mathrm{O}_{2}$ & $\mathrm{CT}$ & AR & WA & E11 & E3 \\
\hline$\#$ & 10 & 78 & 20 & 35 & 1.25 & 47.5 & 22.5 & 7.5 & 7.5 \\
\hline & 10 & 70.1 & 38.4 & 53.4 & 1.25 & 43.4 & 21.5 & 7.5 & 7.5 \\
\hline 10.1 & 130 & 41.9 & 48.1 & 1.25 & 43.4 & 21.5 & 6.22 & 8.78 \\
\hline & 10.4 & 116 & 30.5 & 33.1 & 1.13 & 46 & 22.5 & 6.18 & 8.82 \\
\hline
\end{tabular}

Rule viewer is used to test whether the offspring is suitable for evolve or not. As shown in Table III, the $1^{\text {st }}$ offspring (where the chromosome has 7.5 and 7.5 for defect rate of E11 and E3) is perfectly matched with the output generated by 
rule viewer of MatLab.

The second offspring is not matched with the output generated by rule viewer of MatLab as the offspring value is 10 and 13 respectively but the output generated by rule reviewer for E11 is 6.03 and for E3 is 8.97.

The third offspring (6.18 and 8.7) matches well with the output generated by rule viewer (which is 6.2 for E11 and 8.8 for E3). Similarly, the fourth offspring shown in Table III also shows good match. In total, there are three out of four which were matched within the error rate, the accuracy with error rate is 0.75 .

TABLE III: NEW GENERATED OFFSPRING EXTRACTED FROM THE LATEST POPULATION

\begin{tabular}{|l|l|l|l|l|l|l|l|l|l|l|}
\hline \multicolumn{9}{|c|}{ Input } \\
\hline $\begin{array}{l}\text { Pressure } \\
\text { (PR) }\end{array}$ & $\begin{array}{l}\text { RF } \\
\text { Power(RFP) }\end{array}$ & $\mathrm{CHF}_{3}$ & $\mathrm{O}_{2}$ & $\mathrm{CT}$ & $\mathrm{AR}$ & WA & E11 & E3 \\
\hline 1 & 9.7 & 101 & 24.3 & 44.6 & 1.13 & 46 & 22.5 & $\begin{array}{l}7.5 \\
(7.5)\end{array}$ & $\begin{array}{l}7.5 \\
(7.5)\end{array}$ \\
\hline 2 & 12.2 & 92.5 & 20.8 & 41.1 & 1.35 & 49.7 & 25 & $\begin{array}{l}10 \\
(6.03)\end{array}$ & $\begin{array}{l}13 \\
(8.97)\end{array}$ \\
\hline 3 & 12.2 & 136 & 14.6 & 32.3 & 1.35 & 49.7 & 25 & $\begin{array}{l}6.18 \\
(6.2)\end{array}$ & $\begin{array}{l}8.7 \\
(8.8)\end{array}$ \\
\hline 4 & 11 & 106 & 41.9 & 48.1 & 1.24 & 39.7 & 23.3 & $\begin{array}{l}6 \\
(6.11)\end{array}$ & $\begin{array}{l}8.9 \\
(8.89)\end{array}$ \\
\hline
\end{tabular}

\section{NUMERICAL ANALYSIS}

The numerical analysis in this section is conducted to demonstrate whether the proposed approach is able to achieve the results as expected. The hybrid fuzzy GA approach for RIE process optimization is applied for setting the parameters for the RIE process. There are 37 cases from a magnetic hard disks manufacturer.

The proposed approach was implemented in MatLab. The results of the proposed method were compared with the physical experimental result. The crossover probability for the GA process was set at 0.8 , and the mutation probability for the GA process was set at 0.01. The maximum generations was set at 100 generations. . The GA result shows that fitness value is 0.535 . The results for different numbers of generations are shown in Fig. 3.

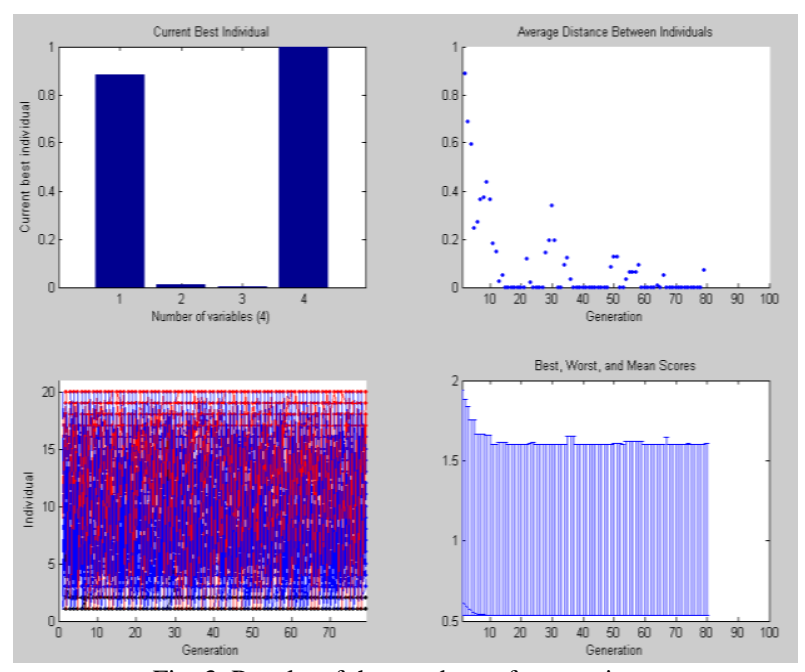

Fig. 3. Results of the numbers of generations.

The maximum etch rate of 38 Angstroms/minute was obtained with the following process parameters and is shown below:

$\begin{array}{llll}\text { RF power (W) } & 99 & \mathrm{CHF}_{3} \text { flow (sccm) } & 30 \\ \text { Pressure (mtorr) } & 10 & \mathrm{O}_{2}(\mathrm{sccm}) & 30\end{array}$

Our results indicates that the process parameter generated by our hybrid Fuzzy GA approach is more than 95 percentage matched with the experiment done by Winnall and Winderbaum (2000). The results show that the proposed approach is able to achieve the results as expected.

\section{CONCLUSIONS}

A hybrid innovative approach has been suggested and tested in a real company to demonstrate its viability in an actual industrial environment. Results indicate that the proposed approach is able to achieve the expected outcomes in an efficient way. In particular, the contribution of this research is the successful introduction of a hybrid approach to identify the optimal criteria for process control in order to achieve optimal performance of the entire operation. The impact of this approach is its characteristic to take advantage of the inherent benefits of the two well-known approaches namely fuzzy logic and GA whilst avoiding the weakness of them with the methodology proposed in the paper.

\section{REFERENCES}

[1] S. Wadhwa, K. S. Bhoon, and F. T. S. Chan, "Postponement strategies through business process redesign in automotive manufacturing," Industrial Management \& Data Systems, vol. 106, no. 3, pp. 307-326, 2006.

[2] S. R. Cheng, B. M. Hsu, and M. H. Shu, "Fuzzy testing and selecting better process performance," Industrial Management \& Data Systems, vol. 107, no. 6, pp. 862-881, 2007.

[3] C. J. Huang, A. J. C. Trappey, and Y. H. Yao, "Developing an agent-based workflow management system for collaborative product design," Industrial Management \& Data Systems, vol. 106, no. 5, pp. 680-699, 2006.

[4] A. C. Caputo, L. Fratocchi, and P. M. Pelagagge, "A genetic approach for freight transportation planning," Industrial Management \& Data Systems, vol. 106, no. 5, pp. 719-738, 2006.

[5] J. Al-Kuzee, T. Matsuura, A. Goodyear, L. Nolle, A. A. Hopgood, P. D. Picton, and N. S. J. Braithwaite, "Optimization of plasma etch processes using evolutionary search methods with in situ diagnostics," Plasma Sources Science Technology, vol. 13, pp. 612-622, 2004.

[6] T. S. Li, C. T. Su, and T. L. Chiang, "Applying robust multi-response quality engineering for parameter selection using a novel neural-genetic algorithm," Computers in Industry, vol. 50, pp. 113-122, 2003.

[7] M. Milfelner, J. Kopac, F. Cus, and U. Zuperl, "Genetic equation for the cutting force in ball-end milling," Journal of Materials Processing Technology, vol. 164-165, pp. 1554-1560, 2005.

[8] P. Chen, M. Taniguchi, and T. Toyota, "Intelligent diagnosis method of multi-fault state for plant machinery using wavelet analysis, genetic programming and possibility theory," in Proc. IEEE International Conference on Robotics and Automation, Taipei, Taiwan, September 14-19, 2003, pp. 610-615.

[9] T. Liu, X. Yang, and G. Kalambur, "Design for machining using expert system and fuzzy logic approach," Journal of Materials Engineering and Performance, vol. 4, pp. 599-609, 1995.

[10] B. Kim and J. H. Park, "Qualitative fuzzy logic model of plasma etching process," IEEE Transactions on Plasma Science, vol. 30, pp. 673-678, 2002.

Henry C. W. Lau was born in 1949 in Hong Kong. He received his master's degree at Aston University in Birmingham in 1981 and his doctorate at the University of Adelaide in 1995.He is currently a senior lecturer of the School of Management at The University of Western Sydney, involving in research and teaching activities. His current research areas cover logistics and supply chain management, operations research, engineering management and artificial intelligence systems. 